\title{
Clinical Efficacy of 50\% Glucose Infusion in Restoring Intradialytic Hypotension
}

\author{
Zhang Xuemei $^{1} \quad$ Huang Shaomin $^{1} \quad$ Lin Lian $^{1}$ Lei Yan $^{1}$ Zhong Xiaochun,"*
}

\begin{abstract}
${ }^{I}$ Department of Nephrology, Center of Nephrology and Urology, The Seventh Affiliated Hospital, Sun Yat-sen University, Shenzhen, China, 518107. Zhang Xuemei, https://www.orcid.org/0000-0003-1509-7485.
\end{abstract}

*Zhong Xiaochun is the corresponding author.Email:zhongxch5@mail.sysu.edu.cn.

Article History Received 9 July 2020 Accepted 25 July 2020 Published 30 September 2020

Cite this Article Zhang Xuemei, Huang Shaomin, Lin Lian, Lei Yan, Zhong Xiaochun. Clinical Efficacy of 50\% Glucose Infusion in Restoring Intradialytic Hypotension [J].Medical Research, 2020.2(3):1-7, http://dx.doi.org/10.6913/MRHK.202009_2(3).0001

Copyright @ 2020 Creative Publishing Co., Limited. All rights reserved. Email:mrhk26640333@ gmail.com.

\begin{abstract}
Objective Intradialytic hypotension complicates up to $30 \%$ of all dialysis treatments and is linked to increased morbidity and mortality. Intravenous fluid infusion is effective to restore blood pressure, yet the optimal replacement fluid remains unclear. This study aimed to evaluate the clinical efficacy of $50 \%$ glucose as the treatment for intradialytic hypotension in maintenance hemodialysis (MHD) patients.

Methods Thirty-five MHD patients with intradialytic hypotension from February 2019 to March 2020 were enrolled in this study. The control group $(\mathrm{n}=17)$ received $100-200 \mathrm{ml}$ normal saline infusion, while the experimental group $(n=18)$ received $100-200 \mathrm{ml} 50 \%$ glucose infusion. The blood pressure before and after hemodialysis was compared to determine the efficacy rate of the treatments.

Results The efficacy rate of the experimental group was significantly higher than that of the control group (efficacy rate: $94.4 \%$ vs $64.7 \%, \mathrm{p}=0.028$ ). Patients in the experimental group restored their blood pressure faster than those in the control group (time to relief of symptoms: $12 \pm 6.5$ vs $7.6 \pm 2.9$ mins, $\mathrm{p}=0.014$ ), indicating that $50 \%$ glucose infusion are more effective than the isotonic saline in treating intradialytic hypotension.
\end{abstract}

Conclusions Compared with isotonic normal saline, $50 \%$ glucose might be a better option for the treatment of hypotension during hemodialysis.

Keywords Intradialytic hypotension; 50\% glucose injection; Clinical efficacy

\section{INTRODUCTION}

Intradialytic hypotension, defined as the presence of a decrease in mean arterial pressure by $10 \mathrm{mmHg}$ or a decrease of systolic pressure $\geqslant 20 \mathrm{mmHg}$, frequently occurs in patients with maintenance hemodialysis (MHD) ${ }^{[1]}$. It complicates $5 \%$ to $30 \%$ of all dialysis treatments ${ }^{[2,3]}$; and it is reported that $75 \%$ of dialysis patients may experience at least one episode of intradialytic hypotension ${ }^{[3,4]}$. MHD patients with repeated 
Xuemei Zhang, Shaomin Huang, et al. Clinical Efficacy of 50\% Glucose Infusion in Restoring Intradialytic Hypotension Medical Research ISSN 2664-0333 eISSN 2664-0341 Volume 2 Issue 3 http://dx.doi.org/10.6913/MRHK.202009_2(3).0001

intradialytic hypotension during dialysis often cause insufficient perfusion to important organs such as brain and heart, increase the risk of internal leakage and cardio-cerebrovascular accidents, and eventually affect the adequacy of hemodialysis and quality of life of patients ${ }^{[4,5]}$. Active prevention and treatment for hemodialysis hypotension can help improve the survival rate and quality of life of patients.

Multiple factors can contribute to intradialytic hypotension, including the rapid change of ultrafiltration rate, a rapid drop in plasma osmolality, incorrectly low prescribed target weight, and cardiac autonomic neuropathy ${ }^{[3]}$. When the ultrafiltration rate is high, intravascular volume depletion exceeds the blood pressure level that can be maintained by the hemodynamic compensation mechanisms, hypotension may occur. In many cases, reducing the ultrafiltration rate or repositioning the patients can restore the blood pressure, depending on the severity of hypotension. However, if blood pressure is not restored, the intravascular volume should be replaced. Intravenous fluid infusion is effective in restoring blood pressure ${ }^{[6]}$. Many clinicians use isotonic normal saline as first-line therapy for hypotension ${ }^{[6,7]}$, while some clinicians use hypertonic glucose, 5\% dextrose, or albumin solutions ${ }^{[8]}$. Previous clinical trials conclude that $5 \%$ albumin is not more effective than normal saline for treating intradialytic hypotension ${ }^{[6,9]}$. However, no studies have been conducted to compare the clinical efficacy of $50 \%$ glucose versus normal saline; and at present, the optimal replacement fluid for intradialytic hypotension remains unknown.

In this randomized trial, we aimed to determine the clinical efficacy of $50 \%$ glucose infusion on intradialytic hypotension among maintenance hemodialysis (MHD) patients. Patients with intradialytic hypotension treated with normal saline were included as the control group.

\section{MATERIALS AND METHODS}

\section{General information}

This randomized controlled trial enrolled 35 MHD adult patients with intradialytic hypotension. These patients received hemodialysis in the Seventh Affiliated Hospital, Sun Yat-sen University from February 2019 to March 2020. The control group ( $n=17)$ received normal saline infusion, while the experimental group received $50 \%$ glucose infusion as treatments for intradialytic hypotension. The baseline information of the included patients was listed in Table 1. Inclusion criteria: (1) >18 years old; (2) maintenance hemodialysis for more than 6 months; (3) diagnosis of hypotension during dialysis; (4) symptoms remained after stopping dehydration, slowing blood flow, or lowering temperature; (5) signed informed consent provided. Exclusion criteria: (1) the blood glucose measured at that time was lower than $3.89 \mathrm{mmol} / 1$; (2) the subject refused; (3) the subject withdrew the consent. This study was conducted following the 1964 Helsinki Declaration. The study protocol was reviewed and approved by the Ethics Committee of The Seventh Affiliated Hospital, Sun Yat-Sen University. All patients or their legal guardians signed the written informed consent documents prior to the study.

\section{Dialysis}

The dialysis machines were Germany Belandega and Campbell's AK-98, the dialysate was bicarbonate, and the dialyzers were loPs-15 and Campbell's 14L. The routine standards of hemodialysis blood flow, 
dialysate flow, ultrafiltration volume, and ultrafiltration rate and dialysis temperature were set at $200-250 \mathrm{~mL} / \mathrm{min}, 500 \mathrm{~mL} / \mathrm{min}, 2.0-3.6 \mathrm{~L}, 500-900 \mathrm{~mL} / \mathrm{min}$ and $36-37^{\circ} \mathrm{C}$ respectively. Three times a week for four hours each time, and the anticoagulant was heparin sodium. The dry weights of the two groups were pre-defined at the time of enrollment and reached the standard for dry mass after adequate hemodialysis. Once hypotension occurs, the blood flow of the both groups should be decreased to $150-200 \mathrm{ml} / \mathrm{min}$, the ultrafiltration rate to less than $300 \mathrm{~mL} / \mathrm{h}$ or be suspended.

\section{Definition of Intradialytic hypotension}

During the dialysis, the systolic blood pressure during dialysis drops by more than $20 \mathrm{mmHg}$ or mean arterial pressure drops by more than $10 \mathrm{mmHg}{ }^{[1]}$, accomplished by obvious nausea and vomiting, chest tightness, dizziness, sweating, fatigue, or loss of consciousness.

\section{Treatments}

The control group was given 100-200ml normal saline, while the experimental group was given $100-200 \mathrm{ml} 50 \%$ glucose. The fluid was given by intravenous drip intravenously from the side tube of the Blood Line Set at maximum speed. The supine blood pressure was measured and recorded every 2 minutes. The supine blood pressure was measured and recorded every 5 minutes. Patients in both groups received some other treatments, such as correction of anemia and hypoproteinemia. No vasopressors or anti-hypertensive drugs were given on the day of dialysis.

\section{Measurements}

The parameters including the occurrence time of hypotension, heart rate, mean arterial pressure, and ultrafiltration volume, and diastolic and systolic blood pressure were measured before and 2 hours after the hemodialysis.

\section{Efficacy evaluation}

The criteria of "significantly effective" and "effective" were listed below. Significantly effective: symptoms of hypotension disappear completely; systolic blood pressure reaches $90 \mathrm{mmHg}$ or above, and MAP rises by $10 \mathrm{mmHg}$ or above after treatments. Effective: symptoms of hypotension are partially relieved; systolic blood pressure reaches $90 \mathrm{mmHg}$ or above, and MAP rises by $0-10 \mathrm{mmHg}$ after treatments. The total efficacy rate of treatments was calculated by the percentage of events that were either "significantly effective" or "effective".

\section{Statistical analysis}

All the data in this study were analyzed with the use of SPSS22.0 software. Measurement data were analyzed using the Student t-test and represented as mean \pm SD. Counting data were analyzed using a $\chi 2$ test and represented as percentages. $P$ values of less than 0.05 were considered significantly different.

\section{RESULT}

\section{Baseline characteristics}

As shown in Table 1, the gender ratio, age (53.8 \pm 6.2 vs $54.2 \pm 6.1$ years), primary diseases, blood 
glucose level, blood pressure before dialysis (systolic: $135.1 \pm 11.8$ vs $130.1 \pm 9.1 \mathrm{mmHg}$ ) and at the time of dialytic hypotension (systolic: $78.1 \pm 5.9 \quad$ vs $78.4 \pm 6.4 \mathrm{mmHg}$ ) were all comparable in patients between the control group and the experimental group. Chronic nephritis was the major primary disease of these patients.

\section{Calculated clinical efficacy rate between the two groups}

The total efficacy rates of the experimental group and the control group were $94.4 \%$ and $64.7 \%$, respectively ( $\mathrm{p}=0.028)$, indicating that $50 \%$ glucose showed a superior effect in restoring the blood pressure during intradialytic hypotension (Table 2). Particularly, 50\% glucose appeared to be "significantly effective" in $55.6 \%$ of patients in the experimental group, while normal saline were "significantly effective" in $41.2 \%$ of controlled group. Moreover, patients in the experimental group restored their blood pressure faster than those in the control group (time to relief of symptoms: $12 \pm 6.5$ vs $7.6 \pm 2.9$ mins, $p=0.014$ ).

Table 1. Baseline characteristics of patients in the control and experimental groups.

\begin{tabular}{ccc}
\hline Parameters & $\begin{array}{c}\text { Control group } \\
(\mathrm{n}=17)\end{array}$ & $\begin{array}{c}\text { Experimental group } \\
(\mathrm{n}=18)\end{array}$ \\
\hline Age (year) & $53.8 \pm 6.2$ & $54.2 \pm 6.1$ \\
Gender (M/F) & $10 / 7$ & $12 / 6$ \\
& Primary diseases & \\
Hypertensive nephropathy & 1 & 2 \\
Obstructive nephropathy & 2 & 3 \\
Chronic nephritis & 10 & 12 \\
Drug-induced renal damage & 2 & 0 \\
Nephrotic syndrome & 2 & 1 \\
& Before dialysis & $130.1 \pm 9.1$ \\
Systolic blood pressure (mmHg) & $135.1 \pm 11.8$ & $87.8 \pm 9.0$ \\
Diastolic blood pressure (mmHg) & $88.4 \pm 9.2$ & \\
Systolic blood pressure (mmHg) & $78.1 \pm 5.9$ & $78.4 \pm 6.4$ \\
Diastolic blood pressure (mmHg) & $46.5 \pm 5.5$ & $44.7 \pm 6.3$ \\
Blood glucose (mmol/L) & $5.1 \pm 1.1$ & $5.2 \pm 1.2$ \\
\hline
\end{tabular}

Table 2. The efficacy rate and time to relief of symptoms in patients receiving saline (Control group) versus $50 \%$ glucose (Experimental group) as treatments.

\begin{tabular}{cccc}
\hline & $\begin{array}{c}\text { Control group } \\
(\mathrm{n}=17)\end{array}$ & $\begin{array}{c}\text { Experimental group } \\
(\mathrm{n}=18)\end{array}$ & $\mathrm{P}$ \\
\hline Significantly effective $(\mathrm{n}, \%)$ & $7(41.2 \%)$ & $10(55.6 \%)$ & $0.028^{\#}$ \\
Effective (n, \%) & $4(23.5 \%)$ & $7(38.9 \%)$ & \\
Ineffective (n, \%) & $6(35.3 \%)$ & $1(5.6 \%)$ & \\
Total efficacy rate & $64.7 \%$ & $94.4 \%$ & $0.014^{*}$ \\
\hline
\end{tabular}

Note: "tested by chi squared $\left(\chi^{2}\right)$ test; ${ }^{*}$ tested by t test.

\section{DISCUSSION}

To date, very few studies addressing the efficacy of glucose infusion as a treatment for intradialytic 
hypotension have been reported; and yet the optimal replacement fluid for patients with intradialytic hypotension remains unclear. Although isotonic normal saline is the most common choice for intradialytic hypotension, it is not effective sometimes in the clinic. This study demonstrated that $50 \%$ glucose infusion showed a higher efficacy rate than the normal saline in restoring the blood pressure of patients during dialysis, suggesting that $50 \%$ glucose could be a better option for the fluid replacement for patients with intradialytic hypotension.

Hemodialysis is a common artificial kidney replacement therapy for acute and chronic renal failure, which can effectively improve patients' disordered internal environment and quality of life to prolong their survival time. However, in practice, dialysis experience is lacking, dialysis equipment is inefficient, and dehydration will reduce the effective circulating blood volume, causing vasoconstriction dysfunction and cardiac output reduction. Therefore, the complications are highly likely to occur, which will adversely affect the patient's dialysis tolerance and dialysis quality ${ }^{[10]}$. Hemodialysis hypotension is one of the common complications of hemodialysis, with an incidence of up to $20 \%-30 \%$, which mainly occurs in cardiovascular diseases, diabetes and the elderly for all of the above patients have left heart failure to a certain extent. The incidence of hypotension during hemodialysis treatment is relatively high, which is related to various factors such as decreased vascular tone, hemodynamic changes, decreased cardiac perfusion, and cardiac diastolic and systolic dysfunction ${ }^{[11]}$. Frequent hemodialysis hypotension will not only lead to inadequate dialysis and increase the risk of internal fistula blockage, but also increase the physical and mental pain of patients and the workload of medical staff.

Related therapies were used in the previous hemodialysis, such as sodium carbonate dialysis, high sodium and low temperature, measurement of dry weight, ultrafiltration rate adjustment based on dry weight, timely corrections of hypoalbuminemia and anemia, the supplement of saline when hypotension occurs. Although these treatments can relieve hemodialysis hypotension to some extent, there is still a high incidence of hemodialysis hypotension in some cases ${ }^{[12-14]}$. $50 \%$ of glucose injection can maintain plasma crystal osmotic pressure, prevent a rapid decrease of plasma crystal osmotic pressure and a downward capillary refill rate caused by the removal of urea and creatinine, reduce the probability of hypotension caused by decreased circulating blood volume, maintain the stability of the internal environment, and ensure the accomplishment of hemodialysis ${ }^{[5,15,16]}$. The results of this study showed that the total efficacy rate of the experimental group was significantly higher than that in the control group $(\mathrm{P}<0.05)$, suggesting that $50 \%$ of glucose injection can significantly improve the clinical efficacy of hemodialysis hypotension, ensure adequate dialysis and improve the quality of dialysis, which is worthy of comprehensive popularization and promotion.

There are several limitations to this study. First, the sample size involved in this study is very small due to the relatively low incidence rate of intradialytic hypotension in our center. Second, this is single center study, so the evidence yielded in this study is not very strong. The randomized clinical trials enrolled patients from multiple centers may be needed to further confirm the superior effectiveness the $50 \%$ glucose. Despite 
Xuemei Zhang, Shaomin Huang, et al. Clinical Efficacy of 50\% Glucose Infusion in Restoring Intradialytic Hypotension Medical Research ISSN 2664-0333 eISSN 2664-0341 Volume 2 Issue 3 http://dx.doi.org/10.6913/MRHK.202009_2(3).0001

these limitations, this study provides the information supporting that $50 \%$ glucose maybe also a good option for patients with intradialytic hypotension.

In summary, although the current clinical hemodialysis technology is relatively advanced and efficient, hemodialysis hypotension is still an urgent problem to be solved, which will pose a threat to the life safety of patients without prompt prevention and treatment. During hemodialysis treatment, it is not only necessary to strictly control the patient's weight, but also to timely correct anemia, improve nutrition and cardiac function, and strengthen individual prevention and treatment based on the actual situation of the patient. In addition, the application of $50 \%$ of glucose injection in hemodialysis hypotension can achieve good therapeutic effects, which may further improve the quality of hemodialysis as well as the quality of life and prognosis of patients.

\section{STATEMENT}

There is no conflict of interest in this article.

\section{REFERENCES}

1 Assimon MM, Flythe JE. Definitions of intradialytic hypotension. Semin Dial. 2017;30(6):464-72.

2 Reilly RF. Attending rounds: A patient with intradialytic hypotension. Clin J Am Soc Nephrol. 2014;9(4):798-803.

3 Sands JJ, Usvyat LA, Sullivan T, Segal JH, Zabetakis P, Kotanko P, et al. Intradialytic hypotension: frequency, sources of variation and correlation with clinical outcome. Hemodial Int. 2014;18(2):415-22.

4 McGuire S, Horton EJ, Renshaw D, Jimenez A, Krishnan N, McGregor G. Hemodynamic Instability during Dialysis: The Potential Role of Intradialytic Exercise. Biomed Res Int. 2018;2018:8276912.

5 Chou JA, Kalantar-Zadeh K, Mathew AT. A brief review of intradialytic hypotension with a focus on survival. Semin Dial. 2017;30(6):473-80.

6 Knoll GA, Grabowski JA, Dervin GF, O'Rourke K. A randomized, controlled trial of albumin versus saline for the treatment of intradialytic hypotension. J Am Soc Nephrol. 2004;15(2):487-92.

7 Emili S, Black NA, Paul RV, Rexing CJ, Ullian ME. A protocol-based treatment for intradialytic hypotension in hospitalized hemodialysis patients. Am J Kidney Dis. 1999;33(6):1107-14.

8 Nette RW, Krepel HP, van den Meiracker AH, Weimar W, Zietse R. Specific effect of the infusion of glucose on blood volume during haemodialysis. Nephrol Dial Transplant. 2002;17(7):1275-80.

9 Yin L, Dubovetsky D, Louzon-Lynch P. Implementation of an Algorithm Utilizing Saline Versus Albumin for the Treatment of Intradialytic Hypotension. Ann Pharmacother. 2019;53(2):159-64.

10 Ghasemi A, Shafiee M, Rowghani K. Stabilizing effects of cool dialysate temperature on hemodynamic parameters in diabetic patients undergoing hemodialysis. Saudi J Kidney Dis Transpl. 2008;19(3):378-83.

11 Dasselaar JJ, de Jong PE, Huisman RM, Franssen CF. Influence of ultrafiltration volume on blood volume changes during hemodialysis as observed in day-of-the-week analysis of hemodialysis sessions. ASAIO J. 2007;53(4):479-84.

12 Lee CT, Hsu CY, Lam KK, Lin CR, Chen JB. Inflammatory markers and hepatocyte growth factor in sustained hemodialysis hypotension. Artif Organs. 2005;29(12):980-3.

13 Nette RW, van den Dorpel MA, Krepel HP, Ie EH, van den Meiracker AH, Poldermans D, et al. Hypotension during hemodialysis results from an impairment of arteriolar tone and left ventricular function. Clin Nephrol. 2005;63(4):276-83. 
14 Kovacic V. [Successful treatment of refractory hypotension during hemodialysis using high levels of sodium in the dialysis solution]. Lijec Vjesn. 2002;124(8-9):255-7.

15 Yalcin AU, Sahin G, Erol M, Bal C. Sertraline hydrochloride treatment for patients with hemodialysis hypotension. Blood Purif. 2002;20(2):150-3.

16 Tomita M, Malhotra D, Dheenan S, Shapiro JI, Henrich WL, Santoro TJ. A potential role for immune activation in hemodialysis hypotension. Ren Fail. 2001;23(5):637-49. 